\title{
Label-Free Quantitative Proteomics To Explore The Action Mechanism of The Pharmaceutical Grade Triticum Vulgare Extract In Speeding Up Keratinocytes Healing.
}

\section{Elva Morretta}

University of Salerno

Antonella D'Agostino

University of Campania "Luigi Vanvitelli"

Elisabetta Cassese

University of Campania "Luigi Vanvitelli"

\section{Barbara Maglione}

Farmaceutici Damor (Italy)

Antonello Petrella

University of Salerno

\section{Chiara Schiraldi}

University of Campania "Luigi Vanvitelli"

Maria Chiara Monti ( $\nabla$ mcmonti@unisa.it)

University of Salerno

\section{Research Article}

Keywords: promoting, HaCat, TVE, modulating

Posted Date: November 18th, 2021

DOI: https://doi.org/10.21203/rs.3.rs-1060580/v1

License: (c) (1) This work is licensed under a Creative Commons Attribution 4.0 International License. Read Full License

Version of Record: A version of this preprint was published at Molecules on February 7th, 2022. See the published version at https://doi.org/10.3390/molecules27031108. 


\section{Abstract}

Plant extracts have shown beneficial properties in skin repair, promoting wound-healing through a plethora of mechanisms. In particular, the poly/oligosaccharidic aqueous extract of Triticum vulgare (TVE), as well as TVE-based products, showed interesting biological assets, fastening wound repair. Indeed, TVE acts in the treatment of tissue regeneration mainly on decubitus and venous leg ulcers. Moreover, on scratched monolayers, TVE prompted HaCat cell migration, correctly modulating the expression of metalloproteases towards a physiological matrix remodeling. Here, using the same HaCat based in vitro scratch model, TVE effect has been investigated thanks to an LFQ proteomic analysis of HaCat secretome and immunoblotting. Indeed, TVE behavior on secreted proteins has not yet been fully deepened and it could be helpful to obtain a comprehensive picture of its bio-pharmacological profile. It is emerged that the TVE treatment induced an up regulation of several proteins in the secretome (to be exact 219) whereas only few were down regulated (to be exact 85). Interestingly, many of the upregulated proteins are implicated in promoting wound-healing related processes such as modulating cellcell interaction and communication, cell proliferation and differentiation and prompting cell adhesion and migration.

\section{Introduction}

The proteome is defined as the set of proteins expressed by an organism and it varies from cell to cell, and it is also altered over time and across different cellular states, for instance after a specific treatment. Nowadays, total cellular proteome can be accurately measured by proteomics-based mass spectrometry (MS). Moreover, in several cases, it seems also interesting to focus the attention on selected smaller sets of proteins of interest (the so-called POIs). POls could be very different, such as those belonging to a chemically-modified subset, to a newly-synthesized one, to a pool of membrane proteins or to a secreted subset of the total proteome. Indeed, the analysis of a more limited proteome fraction has the advantage to identify and quantify those proteins which may be very low abundant against the background of the full proteome ${ }^{1}$. In the research described in this manuscript, which is focalized on the effect of a natural extract on wound healing, it could be straightforward to center the attention on the secretome, which represents all the proteins and cellular machineries that are secreted outside the cell plasma membrane into the extracellular matrix ${ }^{2,3}$. The secretome represents a significant group of proteins, expected to be around $13-20 \%$ of the entire proteome and regulating a plethora of biological and physiological processes, as a relevant supply for therapeutic targets detection ${ }^{4}$. Secreted proteins include cytokines, coagulation factors, growth factors, cell-cell adhesion proteins and other signaling molecules; the secretome is easy to collect and, using opportune precipitation methods, is possible to have samples with an appropriate concentration for proteomic analysis.

In this paper, to study the alterations in HaCat cells secretome, upon scratching, induced by an aqueous extract of Triticum vulgare (TVE), label-free quantified (LFQ) secreted proteins were measured by nanoliquid chromatography coupled to tandem mass spectrometry (nLC-MS/MS, Figure 1), comparing the same sample upon scratching event with and without aqueous TVE. 
Indeed, plant extracts have shown beneficial properties in skin repair, promoting wound-healing through a plethora of mechanisms ${ }^{5}$. Wound healing is a multifaceted process, and it is balanced by several features such as, for instance, the level of damage; it is often linked with the concomitant inflammation process, making wound management a very tricky matter. Recently, the development of agents which speed the tissue wound healing has been of interest with the aim of lessen the therapeutic process ${ }^{6}$. In this scenario, pure bioactive phytochemical constituents such as alkaloids, flavonoids, tannins, saponins and phenols, essential oils or extracts have been tested as wound healing promoting agents due to their pharmacological potential as anti-inflammatory, antioxidant, antibacterial and inducers of pro-collagen synthesis compounds ${ }^{5}$. Among these, the poly/oligosaccharidic aqueous TVE as well as TVE-based products showed interesting biological features, prompting scratch repair through the modulation of specific biomarkers, however a specific study of the secreted proteins has never been reported to the best of our knowledge. TVE acts in the treatment of tissue regeneration mainly on decubitus and venous leg ulcers, sores and burns ${ }^{7,8}$. Literature is recently giving more information on the positive effect of TVE on HaCat cellular model: it has been demonstrated that, on scratched HaCat monolayers, TVE prompted cell migration, correctly modulating the expression of metalloproteases towards physiological matrix remodeling. Furthermore, TVE up-regulated, in the short time, the expression of several biomarkers such as MMP-2, MMP-9, collagen I, elastin, integrin aV and aquaporin 3, thus resulting in an optimal remodeling of dermal tissue during healing ${ }^{9}$. These results were gained using time lapse video microscopy (TLVM), gene expression by RT-PCR and protein quantification by immunoblotting analyses on the whole cell and cell extracts ${ }^{9}$.

The present work goes on with the previous paper by Prof. Schiraldi's group, using the same HaCat based in vitro model in order to deepen TVE effect thanks to LFQ proteomic analysis of the secretome, to shed more lights on the mechanism of action of TVE as an active principle in pharmaceutical preparations for wound treatment (Figure 1). Indeed, as shown by D'Agostino et al. ${ }^{9}$, the most interesting proteins whose expression has been modulated by TVE extract are either secreted, present on cell surface or reside in the extracellular space; thus, we favored secretome analysis to unveil novel targets responsible for the TVE biological outcome. Indeed, to our knowledge, the TVE effect on secreted proteins has not yet been fully deepened and it could be helpful for a comprehensive picture of its bio-pharmacological profile.

We have shown that TVE treatment, in respect of the control, induced an up-regulation of several proteins in the secretome (219) whereas only few are down regulated (85). Interestingly, many of the up-regulated proteins are implicated in promoting wound-healing related processes, such as modulating cell-cell interaction and communication, cell proliferation and differentiation and prompting cell adhesion and migration.

\section{Results}

\section{In Vitro Keratinocytes Scratch Assay by TVE}


Wound healing experiments without addition of FBS, as strictly required for robust secretome analyses, resulted in a slower repair rate when compared to the previously reported data on TVE as an active principle ${ }^{9}$. However, also in this case, it has been evidenced a superior healing when the monolayers were treated with TVE, observing a reduced scratched area at $24 \mathrm{~h}$. Thus, it resulted that TVE was able to stimulate migration and to prompt repair, even in starving condition (Figure 2).

\section{Proteome profiling of HaCat secretomes}

To identify HaCat secretomes proteins, the samples $(n=2)$ were precipitated with trichloroacetic acid and re-dissolved in a denaturing buffer to achieve the optimal concentration for in-solution digestion. The samples were then analyzed by liquid chromatography-tandem mass spectrometry (nanoLC-MS/MS) on a high-resolution mass spectrometer (Q-Exactive Classic) and protein identification was achieved through the Proteome Discoverer software (PD). More in details, MSPepSearch was used to perform a spectral library search and, subsequently, MS/MS spectra were searched by Sequest against a reviewed Homo sapiens database. In total, we identified 2591 proteins common to the two analyzed samples. Then, to assess the quality of our dataset, also gaining functional insights into the identified proteins, we evaluated PD-performed annotation for their Cellular Component, reflecting proteins localization in the cell. As can be observed in Figure 3A, 50\% of PD identified proteins were annotated as being secretome ones, $36 \%$ were annotated as non-secreted ones and around $14 \%$ were un-annotated.

Subsequently, for a deeper analysis of our dataset and to validate PD-obtained results, we performed a gene ontology (GO) enrichment analysis through the DAVID database ${ }^{10,11}$. Briefly, this is a system for hierarchically classifying genes or proteins in a graph structure (i.e., an ontology) retrieving, in a ranked list, both the set of terms used to describe these proteins and the corresponding p-values. We focalized our attention on the Cellular Component terms, for a direct comparison with PD. Thus, Figure 3B graph reports the terms for which at least 100 proteins were retrievable ( $y$-axis) versus their corresponding $p$ Values (x-axis). Only terms showing a $\mathrm{p}$-Value smaller than $10^{-5}$ were selected, as the most confident ones. As can be observed, despite the highest count was obtained for proteins annotated as being cytoplasmic ones, the lowest $p$-Value was by far the one related to proteins annotated to reside in extracellular exosomes, key component of secretomes, thus boosting these proteins to be the most significant ones among all the others.

\section{MS-based quantitative proteomics to disclose alteration of secretomes content by TVE}

Following this initial validation step, we moved to the label-free quantification (LFQ) of the secretomes proteins upon a scratch assay followed by TVE treatment. Three independent secretomes samples deriving from HaCat cells treated or not with TVE were subjected to in solution tryptic digestion followed by high-resolution tandem mass spectrometry (LC-MS/MS) analysis, as previously reported and as depicted in Figure 1. Subsequently, both proteins identification (performed as already described) and 
label-free quantification were achieved through PD, allowing to obtain a list of proteins with their corresponding TVE $v s$ ctrl abundance ratio retrieved merging all the replicates. For each ratio, the corresponding $\mathrm{p}$-Value was also obtained.

In total, we identified and quantified up to 2529 proteins (Supp. Table 1). Next, we compared TVE vs ctrl (Figure 4A) and considered proteins with a p-Value $\leq 0.01$ and a fold change $\geq 2$ or $\leq 0.5$ in 3 out of 3 experiments. This resulted in 219 more abundant proteins corresponding to $8.6 \%$ of the total and 85 less abundant ones corresponding to $3.4 \%$ of the total. We then focused our attention on the proteins whose Gene Ontology "Cellular Component" annotation reported "extracellular" as a key word (Table 1) and, to cluster them, we exploited the kmeans clustering approach of the web tool STRING ${ }^{12}$. The obtained results are shown in Figure 4B (more abundant proteins) and Figure 4C (less abundant proteins). 
Table 1

List of the identified more and less abundant proteins whose "Cellular component" is annotated to be "extracellular". Each protein is reported with its Accession, Gene Name, TVE vs ctrl Abundance Ratio and the related $\mathrm{p}$-Value.

\begin{tabular}{|c|c|c|c|c|}
\hline Accession & $\begin{array}{l}\text { Gene } \\
\text { Symbol }\end{array}$ & Description & $\begin{array}{l}\text { Abundance } \\
\text { Ratio }\end{array}$ & Value \\
\hline 060218 & AKR1B10 & Aldo-keto reductase family 1-member B10 & 100.0000 & 0.0000 \\
\hline Q9GZN4 & PRSS22 & Brain-specific serine protease 4 & 100.0000 & 0.0000 \\
\hline P49662 & CASP4 & Caspase-4 & 100.0000 & 0.0000 \\
\hline 060911 & CTSV & Cathepsin L2 & 100.0000 & 0.0000 \\
\hline Q8NBF2 & NHLRC2 & $\mathrm{NHL}$ repeat-containing protein 2 & 100.0000 & 0.0000 \\
\hline Q9Y5S2 & CDC42BPB & Serine/threonine-protein kinase MRCK beta & 100.0000 & 0.0000 \\
\hline Q6ZMP0 & THSD4 & $\begin{array}{l}\text { Thrombospondin type-1 domain-containing } \\
\text { protein } 4\end{array}$ & 100.0000 & 0.0000 \\
\hline P55081 & MFAP1 & Microfibrillar-associated protein 1 & 14.2020 & 0.0000 \\
\hline A6XMV8 & PRSS2 & Protease serine 2 preproprotein & 7.5430 & 0.0001 \\
\hline Q92876 & KLK6 & Kallikrein-6 & 5.4410 & 0.0012 \\
\hline Q96PD5 & PGLYRP2 & $\mathrm{N}$-acetylmuramoyl-L-alanine amidase & 4.9880 & 0.0001 \\
\hline P23142 & FBLN1 & Fibulin-1 & 4.5230 & 0.0007 \\
\hline Q99519 & NEU1 & Sialidase-1 & 4.5030 & 0.0016 \\
\hline Q13410 & BTN1A1 & Butyrophilin subfamily 1 member A1 & 4.1290 & 0.0007 \\
\hline Q12841 & FSTL1 & Follistatin-related protein 1 & 4.0930 & 0.0056 \\
\hline B4E0K5 & MAPK14 & Mitogen-activated protein kinase & 3.1000 & 0.0629 \\
\hline Q9BYT8 & NLN & Neurolysin, mitochondrial & 3.0870 & 0.0066 \\
\hline Q8NCC3 & PLA2G15 & Phospholipase A2 group XV & 2.7950 & 0.0683 \\
\hline P00742 & F10 & Coagulation factor $\mathrm{X}$ & 2.6100 & 0.0013 \\
\hline P02647 & APOA1 & Apolipoprotein A-I & 2.5110 & 0.1885 \\
\hline P13284 & IFI30 & $\begin{array}{l}\text { Gamma-interferon-inducible lysosomal thiol } \\
\text { reductase }\end{array}$ & 2.3500 & 0.0778 \\
\hline P15086 & CPB1 & Carboxypeptidase B & 2.3180 & 0.3147 \\
\hline P00750 & PLAT & Tissue-type plasminogen activator & 2.2870 & 0.0317 \\
\hline Q9ULZ3 & PYCARD & $\begin{array}{l}\text { Apoptosis-associated speck-like protein } \\
\text { containing a CARD }\end{array}$ & 2.2400 & 0.2064 \\
\hline
\end{tabular}




\begin{tabular}{|lllll|}
\hline Accession & Gene & Description & $\begin{array}{l}\text { Abundance } \\
\text { Ratio }\end{array}$ & $\begin{array}{l}\text { p- } \\
\text { Value }\end{array}$ \\
\hline P14735 & IDE & Insulin-degrading enzyme & 2.1320 & 0.4035 \\
\hline P19827 & ITIH1 & Inter-alpha-trypsin inhibitor heavy chain H1 & 0.4820 & 0.0030 \\
\hline P02794 & FTH1 & Ferritin heavy chain & 0.4730 & 0.0000 \\
\hline Q9NZH8 & IL36G & Interleukin-36 gamma & 0.4710 & 0.2161 \\
\hline P25311 & AZGP1 & Zinc-alpha-2-glycoprotein & 0.4430 & 0.0637 \\
\hline P02748 & C9 & Complement component C9 & 0.4420 & 0.2326 \\
\hline P51884 & LUM & Lumican & 0.4100 & 0.0000 \\
\hline Q92626 & PXDN & Peroxidasin homolog & 0.4000 & 0.0000 \\
\hline Q14508 & WFDC2 & WAP four-disulfide core domain protein 2 & 0.3870 & 0.0104 \\
\hline P18510 & IL1RN & Interleukin-1 receptor antagonist protein & 0.3000 & 0.0073 \\
\hline H0YLF3 & B2M & Beta-2-microglobulin (Fragment) & 0.2000 & 0.0038 \\
\hline P02808 & STATH & Statherin & 0.1660 & 0.0000 \\
\hline P11684 & SCGB1A1 & Uteroglobin & 0.1610 & 0.0000 \\
\hline P0DP57 & SLURP2 & Secreted Ly-6/uPAR domain-containing & 0.1350 & 0.0000 \\
\hline Q04118 & PRB3 & Basic salivary proline-rich protein 3 & 0.0100 & 0.0000 \\
\hline Valid & PntrOU & & \\
\hline
\end{tabular}

\section{Validation trough immunoblotting analysis}

Primary antibodies specific to fibulin 1 (FBLN1), tissue Plasminogen A (tPA), kallikrenin 6 (KLK6) and integrin $\beta 1$ (ITG $\beta 1$ ) were used to carry out immunoblotting analyses for the validation of the proteomic analysis, using GAPDH as a loading normalizer. As shown in Figure 5A-C and in the densitometric analysis in Figure 5D, both the over-expression of fibulin 1, tissue Plasminogen A and kallikrenin 6 induced by TVE were verified and validated. As expected, the signal relative to integrin $\beta 1$, whose concentration didn't change accordingly to proteomic experiments, didn't significative change.

\section{Discussion}

Aging of population is a major concern in western countries, often accompanied by the increase of obesity. In this condition, diabetic foot ulcers are a major issue to phase together with other persistent lesions. Thus, the research and the optimization of potential active principles for new pharmaceutical devices still represent an important challenge ${ }^{13}$. Many medical devices have been presented and are commercially available, as well as natural extracts. In this scenario, the only natural extract registered as pharmaceutical active ingredient is TVE by Farmaceutici Damor S.p.A. Despite the long tradition of use, a 
full characterization in an in vitro model based on the proteomic technologies has never been reported. Among natural compounds, in fact, many plant-derived polysaccharides may play a valuable role although the mechanism of action is still under study for many of them, since they are supposed to be active in a very complex process both in cells and in vivo, involving lots of pathways. In particular, as reported by Romanelli et al. ${ }^{8}$, the aqueous extract of Triticum vulgare (TVE) improves the healing of superficial damaged tissues. Specifically, a reduction in the lesion surface area has been observed; clinical signs (perilesional erythema and bleeding) and symptoms (burning, pain, and itching) of venous leg ulcers were improved by the use of the extract. Moreover, TVE has been tested on the HaCat-supported cell proliferation, correctly modulating the timing of metalloproteases expression towards a consistent and well-assessed matrix remodeling ${ }^{9}$.

In this paper, a quantitative proteomic approach has been applied to TVE treated HaCat cells upon scratch assay. More in details, the secretomes of HaCat cells in presence and in absence of TVE has been explored, identified and quantified. Indeed, in the era of the 'omic'sciences, a quantitative MS-based approach can result very useful to reveal which proteins and, consequently, which pathways are altered by whatever treatment, such as the use of TVE. Our MS-based results were submitted to opportune software analysis to identify the up and down-regulated proteins and a discrete number of the altered ones were validated by immunoblotting analysis, strengthening the proteomic approach.

A considerable part of proteins differentially regulated in the HaCaT secretome in presence of TVE are known to be involved as promoters of cell migration and in cytoskeleton reorganization, two important moments at the base of the wound healing process.

Among these proteins, the serine/threonine-protein kinase MRCK beta (PDB code Q9Y5S2) which has been totally up-regulated by TVE (TVE vs ctr ratio is 100) absolutely takes prominence. Indeed, MRCKa and MRCK $\beta$ (myotonic dystrophy kinase-related Cdc42-binding kinases) belong to a subgroup of Rho GTPase activated serine/threonine kinases acting on the actomyosin cytoskeleton. Together with their role in myosin light chain (MLC) phosphorylation, MRCK isoforms control cell shape and motility. Moreover, they are involved in modulating lamellar actomyosin retrograde flow, crucial to cell protrusion and migration ${ }^{14,15}$. Interestingly, the positive effects of TVE in supporting the repair of skin lesions has been revealed also by the finding of the over expression of thrombospondin type-1 domain-containing protein (TVE vs ctr ratio is 100), microfibrillar-associated protein 1 named MFAP-1 (TVE $v s$ ctr ratio is 14) and fibulin-1 (TVE $v s$ ctr ratio is 4.5 , also validated by immunoblotting), all playing a role in cell adhesion and migration and contributing to the supramolecular organization of extracellular matrix (ECM) architecture. These proteins are extracellular matrix glycoproteins, which take part in microfibril assembly, elastinogenesis and tissue homeostasis ${ }^{16}$. Indeed, microfibrils are composed of several proteins, including fibrillin, fibulins, emilin, thrombospondin, and MFAPs ${ }^{17,18}$, which play numerous roles during the building of the elastic fiber network ${ }^{19}$. Elastic fibers provide firmness, support and flexibility to a wide range of tissues that undergo physiological distension, such as skin, ligaments, blood vessels, nerves, muscles, and bone. Moreover, inhibition of fibulin-1C, one of four fibulin-1 isoforms, reduced proliferation 
and wound healing in human airway smooth muscle cells ${ }^{20}$ and thus an increment in fibulin-1 amount should promote proliferation and wound healing. Furthermore, follistatin-related protein 1, which is 4.1 times up-regulated by TVE, is a secreted glycoprotein involved in various physiological processes, such as angiogenesis, regulation of the immune response, cell proliferation and differentiation. There is still no comprehensive understanding of the spectrum of mechanisms of action of follistatin-like proteins, even if it reported that they promote endothelial cell survival, migration and differentiation into network structures in an AKT-dependent manner ${ }^{21}$. TVE has been shown as a natural agent able to provide skin homeostasis. This feature, among the different dis-regulated protein secreted by human keratinocytes, can be further explained through the up-regulation of Kallikrein 6 (KLK6) with a factor of 5 times, also validated by immunoblotting analysis. In general, KLKs are serine proteases exhibiting trypsin- or chymotrypsin-like activities and sharing important structural and functional properties and they are essential for upholding the epidermal microenvironment. Most of the members of this family are present in skin and regulate skin desquamation and inflammation. KLKs involvement has been proved in skin regeneration and pathology ${ }^{22}$. KLKs have a large variety of substrates including ECM proteins and evidence suggests that they are involved in the different steps of skin wound healing. Focusing of KLK6, Klucky and collaborators showed that its overexpression in human keratinocytes increases proliferation and migration, thanks to mRNA-based assays. The KRK6 molecular mechanism of action involves also Ecadherin: in fact, KLK6 up regulation endorses E-cadherin removal facilitating keratinocyte migration to wrap the site of injury. In line with this, overexpression of KLK6 notably diminishes cell membraneanchored E-cadherin also in mice ${ }^{23}$.

During wound healing, the re-epithelialization phase generally begins with the keratinocytes migration. Next, these cells trigger the differentiation program moving from the basal layer towards the outer surface of the skin ${ }^{24}$. Since these events are notably required for a correct sequence of the phases of tissue repair, we have focused on cathepsin L2, also well known as cathepsin V, which has been found to be fully upregulated in TVE treated samples in line with its role in the regulation of keratinocyte differentiation. Indeed, Cathepsin L2, which is a cysteinyl protease, is deputed to the assembly of collagen fibrils and other multimeric structures involved in skin homeostasis maintenance ${ }^{25}$. In order to make possible keratinocyte relocation upon wounding, the cells must separate by each other to move into the wounded region demanding the remodeling of ECM by its limited proteolytic degradation and by de novo biosynthesis of ECM machinery ${ }^{26-31}$. In the past, ECM degradation, which in turn promotes cell migration, was thought to be exclusively carried out by serine proteases and by matrix metalloproteinases (MMPs) ${ }^{27}$; several studies have shown that cysteine cathepsins are involved in partial hydrolysis of ECM constituents in the extracellular space $29,32,33$. Indeed, the discovery that cathepsins are secreted and remain functionally active outside of the lysosome has indicated new roles for these proteins ${ }^{34}$, since cathepsins in the extracellular matrix are known to participate in physiological processes including wound healing ${ }^{35}$, bone remodeling and processing of pro-hormones. Strictly related to this protein upregulation, the increase of the expression of $\mathrm{Y}$-Interferon-inducible Lysosomal Thiol Reductase (GILT) has been found. Indeed, cathepsins need to be kept in their reduced-active state in the oxidative environment. 
GILT has been the only enzyme described in the endosomes, lysosomes, and phagosomes with the potential to catalyze the reduction of cysteine cathepsins ${ }^{36}$. One of the most interesting proteins which has been 2.3 times up-regulated by TVE is tissue-type plasminogen activator (tPA), a key factor in cellular component movement. Its main function is to switch the abundant, but inactive, zymogen plasminogen to plasmin by hydrolyzing an Arg-Val bond. By controlling this proteolytic step, it plays an important role in tissue remodeling and degradation, in cell migration and many other physio-pathological events. It has been reported that the serine proteases urokinase plasminogen activator and tissue-type plasminogen activator (UPA/tPA) together with the inhibitor PA-1 are finely regulated in several cell types during injury repair ${ }^{37}$. In agreement with the here reported results, we also have found the increased release of carboxypepidase B from HaCaT cells treated with TVE. This enzyme is strictly related to plasminogen system both in the matrix and on the cell surface ${ }^{38}$.

Taken together, these data support the knowledge for which a balanced expression of proteolytic enzymes and their inhibitors in the wound bed is necessary to provide a fine control of focal proteolysis, to facilitate matrix restructuring and cell motility in complex enivironments ${ }^{39}$.

Furthermore, we have also found the 2.5 times overexpression of apolipoprotein A-1 HDL upon TVE treatment. This protein may helpfully influence wound healing by speeding up inflammation resolution and by enhancing re-epithelialization ${ }^{40}$. For instance, in a cellular injury model of neuroblastoma, ApoA 1 was found diminished in terms of expression immediately after the damage, but it definitively increased thereafter, inducing a more rapid healing. Thus, ApoA1 has been increasingly expressed and secreted as a late reply to injury inducing a self-protecting mechanism of the injured system ${ }^{41}$. Other proteins were found to be up-regulated by TVE treatment, which may be involved in wound healing in different ways: for instance trypsin-2 (TAT-2), which can efficiently activate matrix metalloproteinases in vitro ${ }^{42}$; the protein called PYCARD mainly involved in cancer and inflammation progression but also implicated in the recruitment of several pro-resolving factors ${ }^{43-45}$ and the coagulation factor $X$, which takes part in cell migration and proliferation. In general, the coagulation proteases have been suggested to play a role in the pathogenesis of tissue remodeling and fibrosis ${ }^{46}$.

Regarding the protein caspase 4, which is greatly up-regulated by TVE, it has to be said that this protein is commonly known for its apoptotic function even thought, recently, the nonlethal functions of this protein in diverse developmental processes, such as cell differentiation and tissue remodeling have been disclosed. In particular, a phenomenon termed apoptosis-induced compensatory proliferation has been investigated, suggesting the caspase action to be important in wound healing and tissue regeneration ${ }^{47}$.

Concerning the down-regulated proteins, lumican is a dermatan sulfate proteoglycan highly expressed in connective tissue and has the ability to regulate collagen fibril assembly. Previous studies have shown that lumican is involved in wound healing, but the precise effects of lumican on re-epithelialization and wound contraction have not yet been fully understood. For instance, it is reported that lumican can promote skin wound healing by facilitating wound fibroblast activation and contraction but not by 
promoting keratinocyte proliferation and migration ${ }^{48}$. In addition, other studies analyzed the effect of chondroitin variants on keratinocytes response and confirmed that sulfation pattern has a role on migration proliferation, and protein expression and assembly in the ECM. In fact, the sulfation degree of glucosaminoglycans affected different biological processes like the interaction with growth factors, cytokines, chemokines, adhesion molecules and lipo-proteins ${ }^{49}$. Our result is one of the first one obtained from a proteomic study reporting a disregulation of lumican in keratinocytes secretome. Thus, it surely appears as appealing element to deepen the investigation about the role of this protein in keratinocytes and, in particular, in the cross-talk between these cells and ECM, during tissue repair. Another downregulated protein revealed in this work has been the ferritin, the major intracellular iron storage protein essential for maintaining the cellular redox status. Interestingly, in recent years, wound healing assays were performed to measure migration capacity of cells with and without ferritin: it has been reported that the silencing of ferritin expression promotes cellular migration, in fully agreement with our data ${ }^{50}$. Furthermore, it has been found as down-regulated the protein interleukin-36 gamma (IL-36y), a member of the IL-1 family of cytokines which act through a common receptor. The role of ILs-36 has been demonstrated extensively in the skin, where they can act on keratinocytes and immune cells to induce a robust inflammatory response. In vitro experiments have shown that ILs-36 bind to IL-36 receptor in keratinocytes and macrophages and releases cytokines and chemokines. Thus, in case of an increased expression of IL-36y in the injured skin, a massive inflammatory stimulus could be triggered on neutrophils, macrophages, and keratinocytes ${ }^{51,52}$. In our experiments, the down-regulation of IL-36Y seems to agree with a shutdown of inflammation, a crucial step for the tight follow-up of skin wound healing. Finally, the protein called secreted Ly-6/UPAR domain-containing protein 2 (SLURP2) is reported to regulate keratinocytes proliferation, differentiation and apoptosis. Members of the lymphocyte antigen6 (Ly6)/urokinase-type plasminogen activator receptor (UPAR) superfamily of proteins are cysteine-rich proteins characterized by a distinct disulfide bridge pattern that creates the three-finger Ly6/uPAR (LU) domain ${ }^{53}$. The SLURP2 is a gene strongly induced in psoriatic skin lesions, but the mechanisms controlling SLURP2 expression are largely unknown, so it still needs to be investigated.

\section{Conclusions}

The quantitative proteomics analysis of HaCat secretomes treated or not with TVE threw further light on its mechanism of action. In particular, the ability of this natural polysaccharide to induce the biosynthesis or the release of specific proteins from keratinocytes makes even more attractive its use in the treatment of skin lesions. In turn, the majority of secreted proteins found to be altered by TVE have been recognized as effectors in cells crosstalk, which plays a central role in the repair of a tissue damage and in the regeneration of diverse cell types. Finally, our results pave a fascinating way for new modalities of investigation on the mechanism of action of products commonly used in the wound repair or even which are still subject of research in this field.

\section{Methods}




\section{Cell Viability Assay}

A human keratinocyte cell line (HaCaT) (purchased from Istituto Zooprofilattico, Brescia, Italy), were cultured in Dulbecco's Modified Eagle Medium (DMEM) supplemented with 10\% (v/v) heat inactivated fetal bovine serum (FBS), $100 \mathrm{U} / \mathrm{mL}$ penicillin and $100 \mu \mathrm{g} / \mathrm{mL}$ streptomycin. All materials were purchased from Gibco (Thermo Fisher scientific, Italy). The cells were grown on tissue culture plates (Corning Incorporated, New York, USA), using an incubator with a humidified atmosphere ( $95 \%$ air $\left./ 5 \% \mathrm{CO}_{2} \mathrm{v} / \mathrm{v}\right)$ at $37^{\circ} \mathrm{C}$. Collagen was purchased from Sigma Aldrich (Milan, Italy). For the experiment $5 \times 10^{4} \mathrm{cell} / \mathrm{cm}^{2}$ were seeded 6 culture plates (Corning, $100 \mathrm{~mm}$ diameter), previously coated with collagen $0.1 \mathrm{mg} / \mathrm{ml}$ in $\mathrm{CH}_{3} \mathrm{COOH}(2 \% \mathrm{v} / \mathrm{v})$, with immortalized keratinocytes (HaCaT) until complete confluence after $48 \mathrm{~h}$.

\section{In Vitro Keratinocytes Scratch Assay by TVE}

The keratinocyte monolayer has been scratched to mimic an in vitro wound, successively the cell monolayers have been washed with sterile PBS for three times to completely remove the debris and FBS residues and added DMEM without serum in the control (triplicates) and in the treatment's wells where $5 \%$ $\mathrm{v} / \mathrm{v}$ Triticum vulgare extract was added to the medium (from Damor Farmaceutici) (triplicates). After $24 \mathrm{~h}$ the supernatants have been recovered.

\section{HaCat secretomes sample preparation for proteomics analysis}

HaCat control and TVE secretomes (three biological replicates) were precipitated with trichloroacetic acid (TCA, final concentration $25 \% \mathrm{vol} / \mathrm{vol}$ in $12 \mathrm{~mL}$ ) overnight at $4^{\circ} \mathrm{C}$ under continuous shaking.

Subsequently, samples were centrifuged $\left(21000 \mathrm{rcf}, 30 \mathrm{~min}\right.$ at $\left.4^{\circ} \mathrm{C}\right)$ and supernatants were discarded. Pellets were then washed with $1 \mathrm{~mL}$ of ice-cooled acetone and centrifuged. These operations were repeated twice. The obtained pellets were re-suspended in $80 \mu \mathrm{L}$ of $8 \mathrm{M}$ Urea/50 mM AmBic (pH 8.5), protein concentration was evaluated through Bradford Assay (Bio-Rad, Hercules, CA, USA) and then equal protein amounts $(50 \mu \mathrm{g}$ in $60 \mu \mathrm{L})$ were submitted to in solution digestion. Briefly, proteins were reduced with $10 \mathrm{mM}$ 1,4-dithiothreitol (DTT, $1 \mathrm{~h}, 25^{\circ} \mathrm{C}, 800 \mathrm{rpm}$ ) and the formed thiols were carboxyamidomethylated with $20 \mathrm{mM}$ iodoacetamide (IAA, $30 \mathrm{~min}, 25^{\circ} \mathrm{C}, 800 \mathrm{rpm}$, in the dark), whose excess was then quenched with $10 \mathrm{mM}$ DTT $\left(10 \mathrm{~min}, 25^{\circ} \mathrm{C}, 800 \mathrm{rpm}\right)$. Urea was then diluted to $1 \mathrm{M}$ with $50 \mathrm{mM}$ AmBic and a trypsin/LysC solution (Promega, Madison, Wisconsin) was added at the enzyme to proteins ratio of $1: 100 \mathrm{w} / \mathrm{w}$ overnight at $37^{\circ} \mathrm{C}$ under continuous shaking. The enzymes were then quenched adding formic acid (FA) to lower the $\mathrm{pH}$ to 3 and the peptides mixture was dried under vacuum, dissolved in 5\% FA and equal amounts aliquots were desalted through C18 ZipTips (ZipTip® Pipette Tips, Merck Millipore Ltd, Cork, Ireland), as reported by manufactures. The obtained samples were dried under vacuum and dissolved in 10\% FA for the subsequent nano-UPLC-MS/MS analysis.

\section{Proteomics analysis: nano-UPLC-MS/MS}

Control and TVE secretomes digests ( $1 \mu \mathrm{g}$ each) were analyzed on an Orbitrap Q-Exactive Classic Mass Spectrometer (ThermoFisher Scientific, Bremen) coupled to an UltiMate 3000 Ultra-High Pressure Liquid 
Chromatography (UPLC) system (ThermoFisher Scientific, Bremen), equipped with an EASY-Spray PepMAPTM RSLC C18 column ( $3 \mu \mathrm{m}, 100 \AA, 75 \mu \mathrm{m} \times 50 \mathrm{~cm}$, ThermoFisher Scientific, Bremen). Peptides elution was achieved at a flow rate of $300 \mathrm{~nL} / \mathrm{min}$ with the following gradient: 1 min at $3 \% \mathrm{~B}, 1 \mathrm{~min}-120$ min to $38 \%$ B, 120 min-121 min to $80 \%$ B, 121 min-131 min at $80 \%$ B, 131 min-132 min back to $3 \%$, until 140 min (A: $\left.0.1 \% \mathrm{AcOH}, 95 \% \mathrm{H}_{2} \mathrm{O}, 5 \% \mathrm{ACN} ; \mathrm{B}: 0.1 \% \mathrm{AcOH}, 95 \% \mathrm{ACN}, 5 \% \mathrm{H}_{2} \mathrm{O}\right)$. The mass spectrometer was operated in data-dependent acquisition mode. Full scan MS spectra were acquired with the following settings: scan range $375-1500 \mathrm{~m} / \mathrm{z}$, full-scan automatic gain control (AGC) target $3 \mathrm{e} 6$ at 70000 resolution, maximum injection time $80 \mathrm{~ms}$. MS2 spectra were generated for up to 6 precursors (normalized collision energy of $28 \%$ ) and the fragment ions acquired at a resolution of 17500 with an AGC target of $1 \mathrm{e} 5$ and a maximum injection time of $110 \mathrm{~ms}$.

\section{Data processing through Proteome Discoverer}

All the .raw MS files were searched with the Proteome Discoverer software (version 2.4.1.15). MSPepSearch was used to perform a spectral library search (NIST Human Orbitrap HCD Library, 1127970 spectra, September 2016) with a mass tolerance of $10 \mathrm{ppm}$ for MS1 and 0.02 Da for MS2. The target False Discovery Rate (FDR) were set to 1\% (strict) and 5\% (relaxed). Subsequently, MS/MS spectra were also searched by Sequest against a reviewed Homo sapiens database (SwissProt, November 2020, 20380 entries) using the following parameters: trypsin digestion; maximum of two missed cleavages; cysteine carbamidomethylation as fixed modification; methionine oxidization, protein $\mathrm{N}$-terminal acetylation and/or de-methylation as variable modifications. Mass tolerances and FDR were set as previously reported. Label-free quantification was performed exploiting both unique and razor peptides for proteins abundance calculation and a pairwise ratio-based approach was used to evaluate TVE vs ctrl proteins abundance. For each calculated ratio, a background-based t-test was performed.

\section{Data visualization}

Proteome Discoverer identified proteins were represented through GraphPad Prism 7.0 as a Volcano Plot, plotting each protein $-\log _{10} p$-value versus the related $\log _{2}(T V E / c t r l)$ fold change. Subsequently, protein clustering was performed through the web tool STRING using a 0.2 confidence and applying a kmeans cluster approach to 4 clusters.

\section{Immunoblotting analysis}

Aliquots of the same TVE-treated and ctrl samples of the different biological replicates exploited for the MS analysis were pooled together, and $10 \mu \mathrm{g}$ of proteins for each pool were treated with Laemmli buffer (60 mM Tris-HCl pH 6.8, 2\% SDS, 0.001\% bromophenol blue, 1\% glycerol, 2\% $\beta$-mercaptoethanol) and heated for 5 minutes at $95^{\circ} \mathrm{C}$. The samples were then loaded into a $12 \%$ polyacrylamide gel for the monodimensional electrophoresis and the subsequently resolved proteins were transferred onto a nitrocellulose membrane. The membrane was soaked for $1 \mathrm{~h}$ at room temperature in a $5 \%$ non-fat dried milk containing TBS-t solution ( $31 \mathrm{mM}$ Tris pH 8, $170 \mathrm{mM} \mathrm{NaCl}, 3.35 \mathrm{mM} \mathrm{KCl}, 0.05 \%$ Tween 20) and then incubated overnight at $4^{\circ} \mathrm{C}$ with either a monoclonal primary anti-body against fibulin 1 (FBLN1, mouse, 1:500 vol/vol, sc-25281, Santa Cruz Biotechnology, Inc., Dallas, TX, USA), tissue Plasminogen A (tPA, 
1:250 vol/vol, sc-69740, Santa Cruz Biotechnology, Inc., Dallas, TX, USA), kallikrenin 6 (KLK6, 1:250 vol/vol, sc-374564, Santa Cruz Biotechnology, Inc., Dallas, TX, USA) and integrin $\beta 1$ (ITG $\beta 1,1: 1000$ $\mathrm{vol} / \mathrm{vol}, 34971$, Cell Signaling Technologies, Danvers, Massachusetts), all in milk/TBS-t. After recognition of the primary antibodies from the proper HPR-tagged secondary ones (1:500 vol/vol mouse for FBLN1, tPA and KLK6 and 1:1500 vol/vol rabbit for ITG $\beta 1$ ), chemiluminescence, developed upon treatment of the membrane with a mixture of luminol and hydrogen peroxide, was detected using an ImageQuant LAS4000 imaging system (GE Healthcare, Waukesha, WI, USA). The densitometric analyses were elaborated through ImageJ. Normalized densitometric values were calculated based on the signals obtained by hybridizing the membranes with a monoclonal antibody against GAPDH (Invitrogen by Thermo Scientific, 1: $1500 \mathrm{vol} / \mathrm{vol})$. Immunoblotting analyses were repeated twice.

\section{Declarations}

\section{Acknowledgements}

We thank Damor Farmaceutici S.p.A for kindly providing TVE and for helpful discussion.

\section{Fundings}

This research was funded by Financial support from INVITALIA-Italy, under Grant CDS 000463.

\section{Author contributions statement}

Conceptualization, M.C.M. B.M. and C.S.; investigation, E.M., A.A, E.C.; resources, B.M. and M.C.M.; data curation, E.M, A.P., M.C.M.; writing-original draft preparation, A.P, M.C.; writ-ing-review and editing, E.M, A.P, A.A, C.S, M.C.M; funding acquisition, M.C.M. All authors have read and agreed to the published version of the manuscript.

\section{Conflicts of Interest}

The authors declare no conflict of interest.

\section{References}

1. Kleinpenning, F., Steigenberger, B., Wu, W. \& Heck, A. J. R. Fishing for newly synthesized proteins with phosphonate-handles. Nat. Commun. 11, 3244 (2020).

2. Tjalsma, H., Bolhuis, A., Jongbloed, J. D. H., Bron, S. \& van Dijl, J. M. Signal Peptide-Dependent Protein Transport in Bacillus subtilis: a Genome-Based Survey of the Secretome. Microbiol. Mol. Biol. Rev. 64, 515-547 (2000).

3. McCotter, S. W., Horianopoulos, L. C. \& Kronstad, J. W. Regulation of the fungal secretome. Curr. Genet. 62, 533-545 (2016). 
4. Mukherjee, P. \& Mani, S. Methodologies to decipher the cell secretome. Biochim. Biophys. Acta Proteins Proteomics 1834, 2226-2232 (2013).

5. Ibrahim, N. et al. Wound Healing Properties of Selected Natural Products. Int. J. Environ. Res. Public Health 15, 2360 (2018).

6. Harper, D., Young, A. \& McNaught, C.-E. The physiology of wound healing. Surg. 32, 445-450 (2014).

7. Funel, N. et al. Triticum vulgare Extract Modulates Protein-Kinase B and Matrix Metalloproteinases 9 Protein Expression in BV-2 Cells: Bioactivity on Inflammatory Pathway Associated with Molecular Mechanism Wound Healing. Mediators Inflamm. 2020, 1-13 (2020).

8. Romanelli, M. et al. Clinical evaluation of the efficacy and safety of a medical device in various forms containing Triticum vulgare for the treatment of venous leg ulcers \&amp;ndash; a randomized pilot study. Drug Des. Devel. Ther. 9, 2787 (2015).

9. D'Agostino, A. et al. Molecular Mechanisms at the Basis of Pharmaceutical Grade Triticum vulgare Extract Efficacy in Prompting Keratinocytes Healing. Molecules 25, 431 (2020).

10. Dennis, G. et al. DAVID: Database for Annotation, Visualization, and Integrated Discovery. Genome Biol. 4, (2003).

11. Huang, D. W. et al. DAVID Bioinformatics Resources: expanded annotation database and novel algorithms to better extract biology from large gene lists. Nucleic Acids Res. 35, W169-W175 (2007).

12. Szklarczyk, D. et al. The STRING database in 2017: Quality-controlled protein-protein association networks, made broadly accessible. Nucleic Acids Res. 45, D362-D368 (2017).

13. Belvedere, R. et al. The promising pro-healing role of the association of mesoglycan and lactoferrin on skin lesions. Eur. J. Pharm. Sci. 163, 105886 (2021).

14. Tan, I., Lai, J., Yong, J., Li, S. F. Y. \& Leung, T. Chelerythrine perturbs lamellar actomyosin filaments by selective inhibition of myotonic dystrophy kinase-related Cdc42-binding kinase. FEBS Lett. 585, $1260-$ 1268 (2011).

15. Heikkila, T. et al. Co-Crystal Structures of Inhibitors with MRCK $\beta$, a Key Regulator of Tumor Cell Invasion. PLoS One 6, e24825 (2011).

16. Mecham, R. P. \& Gibson, M. A. The microfibril-associated glycoproteins (MAGPs) and the microfibrillar niche. Matrix Biol. 47, 13-33 (2015).

17. Rosenbloom, J., Abrams, W. R. \& Mecham, R. Extracellular matrix 4: The elastic fiber. FASEB J. 7, 1208-1218 (1993). 
18. Wagenseil, J. E. \& Mecham, R. P. New insights into elastic fiber assembly. Birth Defects Res. Part C Embryo Today Rev. 81, 229-240 (2007).

19. Zhu, S. et al. Molecular structure and function of microfibrillar-associated proteins in skeletal and metabolic disorders and cancers. J. Cell. Physiol. 236, 41-48 (2021).

20. Ge, Q. et al. Fibulin1C peptide induces cell attachment and extracellular matrix deposition in lung fibroblasts. Sci. Rep. 5, 9496 (2015).

21. Parfenova, O. K., Kukes, V. G. \& Grishin, D. V. Follistatin-Like Proteins: Structure, Functions and Biomedical Importance. Biomedicines 9, 999 (2021).

22. Nauroy, P. \& Nyström, A. Kallikreins: Essential epidermal messengers for regulation of the skin microenvironment during homeostasis, repair and disease. Matrix Biol. Plus 6-7, 100019 (2020).

23. Klucky, B. et al. Kallikrein 6 Induces E-Cadherin Shedding and Promotes Cell Proliferation, Migration, and Invasion. Cancer Res. 67, 8198-8206 (2007).

24. Bizzarro, V. et al. Mesoglycan induces keratinocyte activation by triggering syndecan-4 pathway and the formation of the annexin A1/S100A11 complex. J. Cell. Physiol. 234, 20174-20192 (2019).

25. Berdowska, I. Cysteine proteases as disease markers. Clin. Chim. Acta 342, 41-69 (2004).

26. P, M. Wound healing-aiming for perfect skin regeneration. Science 276, 75-81 (1997).

27. Singer, A. J. \& Clark, R. A. F. Cutaneous Wound Healing. N. Engl. J. Med. 341, 738-746 (1999).

28. Friedl, P. Prespecification and plasticity: shifting mechanisms of cell migration. Curr. Opin. Cell Biol. $16,14-23$ (2004).

29. Everts, V., van der Zee, E., Creemers, L. \& Beertsen, W. Phagocytosis and intracellular digestion of collagen, its role in turnover and remodelling. Histochem. J. 28, 229-245 (1996).

30. Murphy, G. \& Gavrilovic, J. Proteolysis and cell migration: creating a path? Curr. Opin. Cell Biol. 11, 614-621 (1999).

31. Wolf, K. \& Friedl, P. Functional imaging of pericellular proteolysis in cancer cell invasion. Biochimie 87, 315-320 (2005).

32. Eeckhout, Y. \& Vaes, G. Further studies on the activation of procollagenase, the latent precursor of bone collagenase. Effects of lysosomal cathepsin B, plasmin and kallikrein, and spontaneous activation. Biochem. J. 166, 21-31 (1977).

33. Guinec, N., Dalet-Fumeron, V. \& Pagano, M. "In vitro" Study of Basement Membrane Degradation by the Cysteine Proteinases, Cathepsins B, B-Like and L. Digestion of Collagen IV, Laminin, Fibronectin, and 
Release of Gelatinase Activities front Basement Membrane Fibronectin. Biol. Chem. Hoppe. Seyler. 374, 1135-1146 (1993).

34. Yadati, T., Houben, T., Bitorina, A. \& Shiri-Sverdlov, R. The Ins and Outs of Cathepsins: Physiological Function and Role in Disease Management. Cel/s 9, 1679 (2020).

35. Rünger, T. M., Quintanilla-Dieck, M. J. \& Bhawan, J. Role of Cathepsin K in the Turnover of the Dermal Extracellular Matrix during Scar Formation. J. Invest. Dermatol. 127, 293-297 (2007).

36. Balce, D. R., Allan, E. R. O., McKenna, N. \& Yates, R. M. Y-Interferon-inducible Lysosomal Thiol Reductase (GILT) Maintains Phagosomal Proteolysis in Alternatively Activated Macrophages. J. Biol. Chem. 289, 31891-31904 (2014).

37. Ghosh, A. K. \& Vaughan, D. E. PAl-1 in tissue fibrosis. J. Cell. Physiol. 227, 493-507 (2012).

38. Guimarães, A. H. C. et al. TAFI and Pancreatic Carboxypeptidase B Modulate In Vitro Capillary Tube Formation by Human Microvascular Endothelial Cells. Arterioscler. Thromb. Vasc. Biol. 27, 2157-2162 (2007).

39. Simone, T. M. et al. SERPINE1: A Molecular Switch in the Proliferation-Migration Dichotomy in Wound-"Activated" Keratinocytes. Adv. Wound Care 3, 281-290 (2014).

40. Gordts, S., Muthuramu, I., Amin, R., Jacobs, F. \& De Geest, B. The Impact of Lipoproteins on Wound Healing: Topical HDL Therapy Corrects Delayed Wound Healing in Apolipoprotein E Deficient Mice. Pharmaceuticals 7, 419-432 (2014).

41. Sengupta, M. B., Saha, S., Mohanty, P. K., Mukhopadhyay, K. K. \& Mukhopadhyay, D. Increased expression of ApoA1 after neuronal injury may be beneficial for healing. Mol. Cell. Biochem. 424, 45-55 (2017).

42. Nyberg, P. et al. MMP-9 Activation by Tumor Trypsin-2 Enhances in vivo Invasion of Human Tongue Carcinoma Cells. J. Dent. Res. 81, 831-835 (2002).

43. Guo, X. \& Dhodapkar, K. M. Central and overlapping role of Cathepsin B and inflammasome adaptor ASC in antigen presenting function of human dendritic cells. Hum. Immunol. 73, 871-878 (2012).

44. Taxman, D. J. et al. The NLR Adaptor ASC/PYCARD Regulates DUSP10, Mitogen-activated Protein Kinase (MAPK), and Chemokine Induction Independent of the Inflammasome. J. Biol. Chem. 286, 1960519616 (2011).

45. Sartoretto, S. et al. Apoptosis-associated speck-like protein containing a caspase-1 recruitment domain (ASC) contributes to osteoblast differentiation and osteogenesis. J. Cell. Physiol. 234, 41404153 (2019). 
46. Hoffman, M. The multiple roles of tissue factor in wound healing. Front. Biosci. S4, 295 (2012).

47. Fuchs, Y. \& Steller, H. Programmed Cell Death in Animal Development and Disease. Cell 147, 742758 (2011).

48. Liu, X.-J. et al. Lumican Accelerates Wound Healing by Enhancing a2 $\beta 1$ Integrin-Mediated Fibroblast Contractility. PLoS One 8, e67124 (2013).

49. Corsuto, L. et al. Sulfation degree not origin of chondroitin sulfate derivatives modulates keratinocyte response. Carbohydr. Polym. 191, 53-64 (2018).

50. Lobello, N. et al. Ferritin heavy chain is a negative regulator of ovarian cancer stem cell expansion and epithelial to mesenchymal transition. Oncotarget 7, 62019-62033 (2016).

51. Foster, A. M. et al. IL-36 Promotes Myeloid Cell Infiltration, Activation, and Inflammatory Activity in Skin. J. Immunol. 192, 6053-6061 (2014).

52. Dietrich, D. et al. Interleukin-36 potently stimulates human M2 macrophages, Langerhans cells and keratinocytes to produce pro-inflammatory cytokines. Cytokine 84, 88-98 (2016).

53. Loughner, C. L. et al. Organization, evolution and functions of the human and mouse Ly6/uPAR family genes. Hum. Genomics 10, 10 (2016).

\section{Figures}

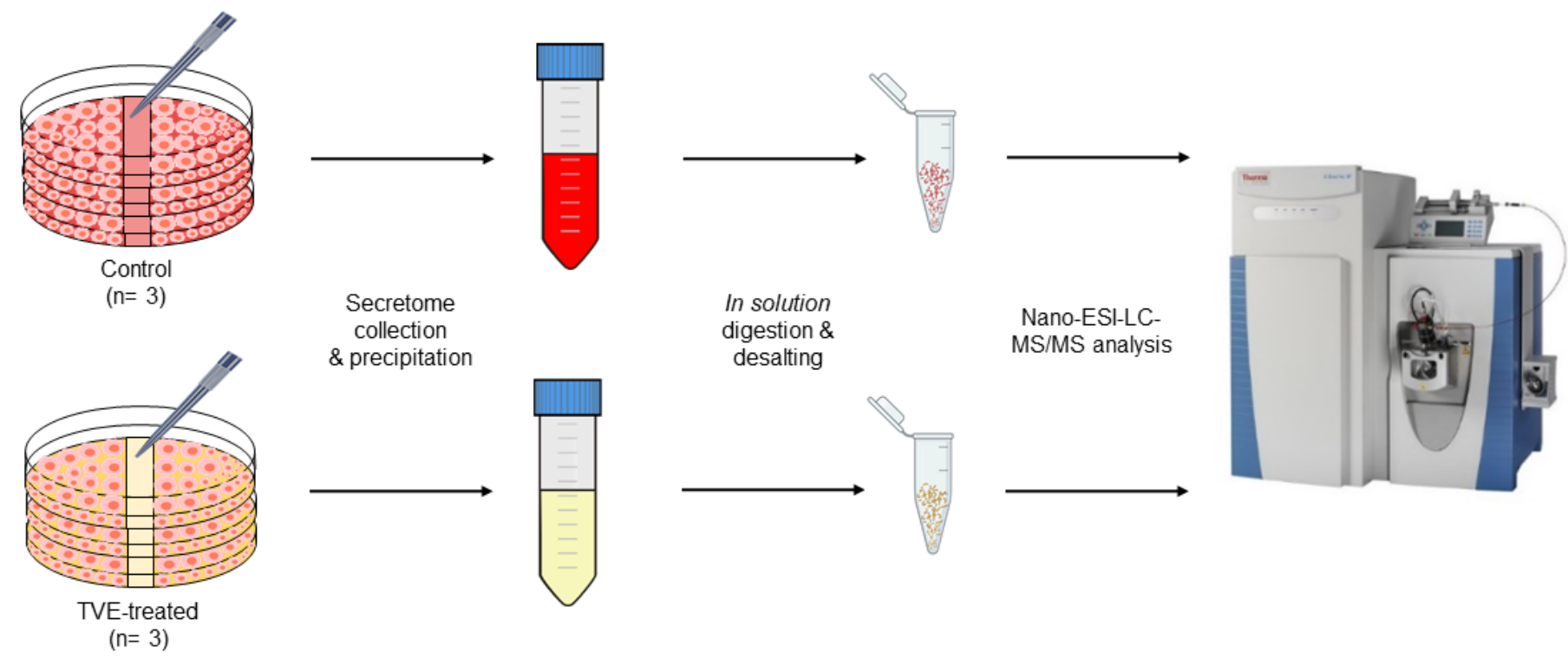

\section{Figure 1}


Experimental workflow.

CTR

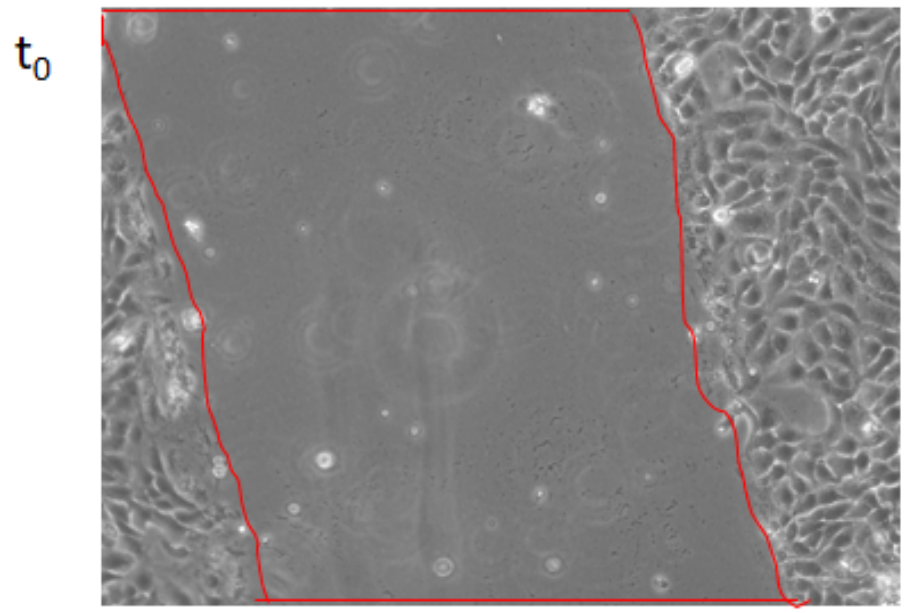

$t_{24 h}$

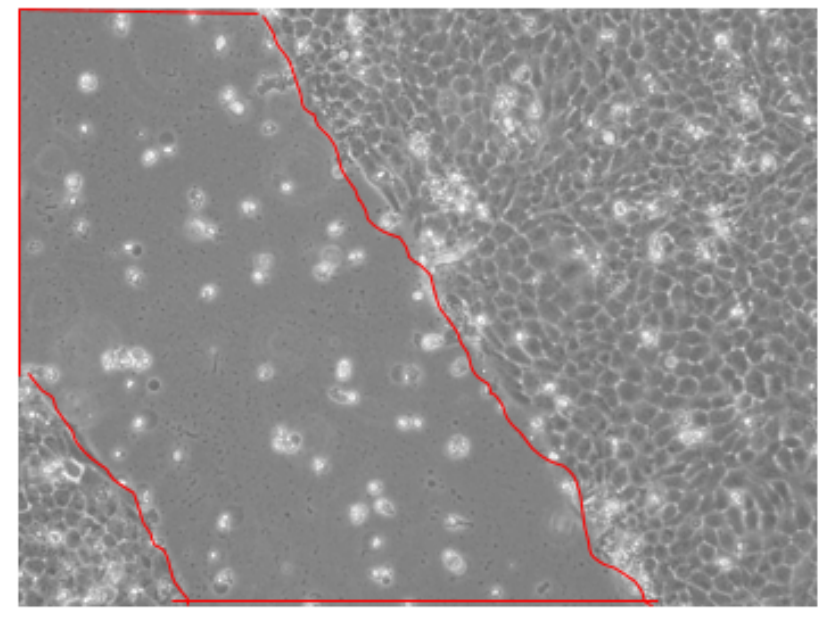

TVE 5\%
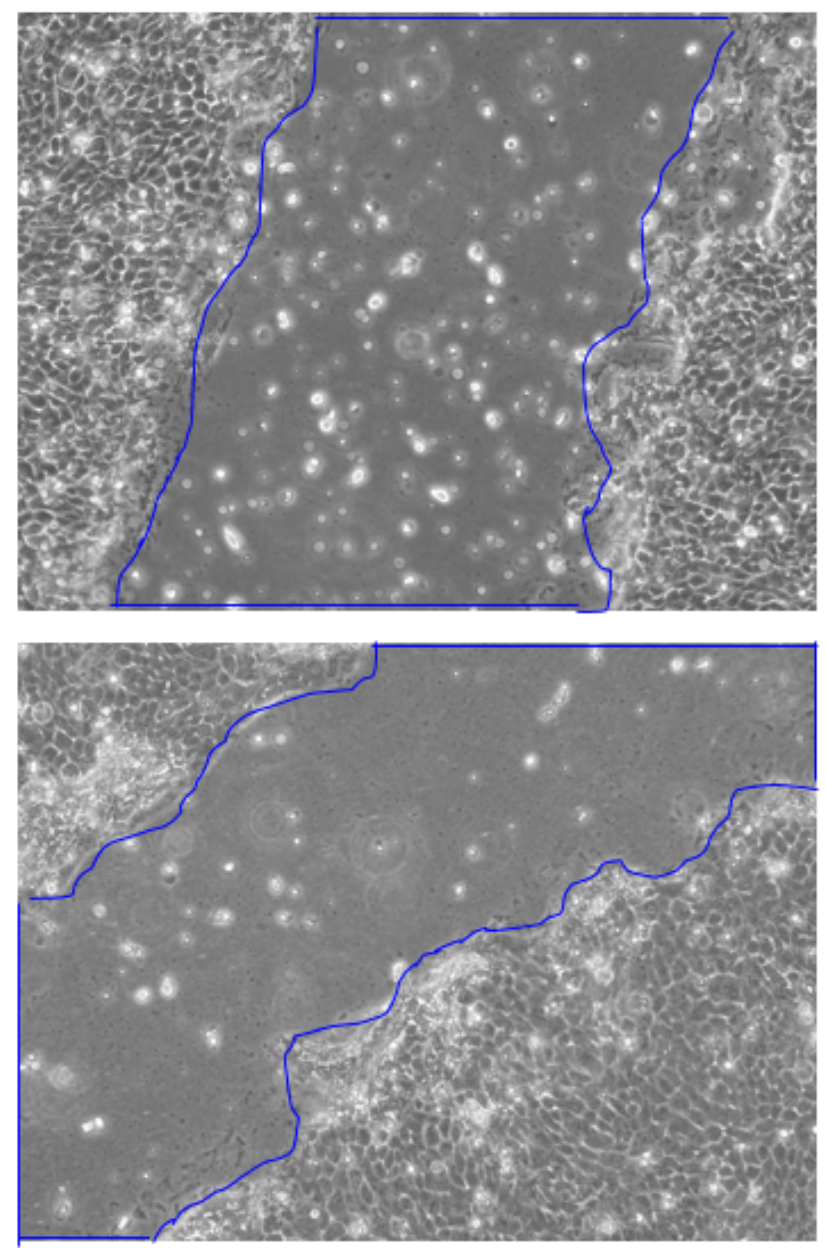

\section{Figure 2}

Representative micrographs of scratched keratinocytes monolayer treated or not with $5 \% \mathrm{~V} / \mathrm{v}$ of TVE, captured at optical microscope (magnification $10 \mathrm{X}$ ) at time 0 and $24 \mathrm{~h}$. 
A

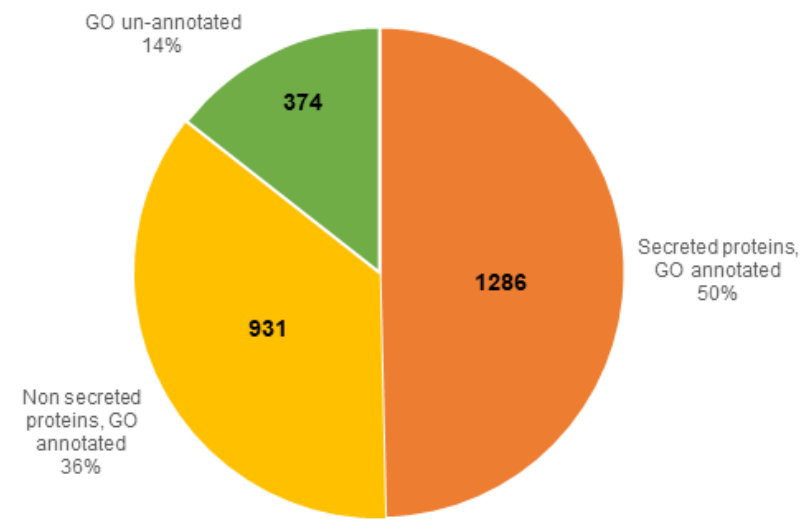

B

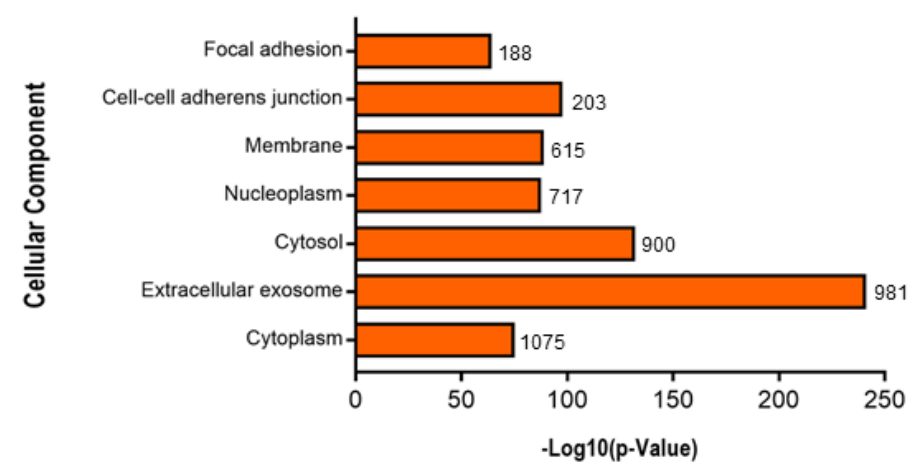

\section{Figure 3}

(A) Pie-chart of PD Cellular Component annotation of proteins identified in two secretome samples. (B) $\mathrm{GO}$ annotation of the Cellular Component terms of all the identified proteins versus their $\mathrm{p}$-Values, performed using the DAVID database and the full Homo sapiens proteome as a background. For each term, the corresponding protein count is reported next to the bar.

A

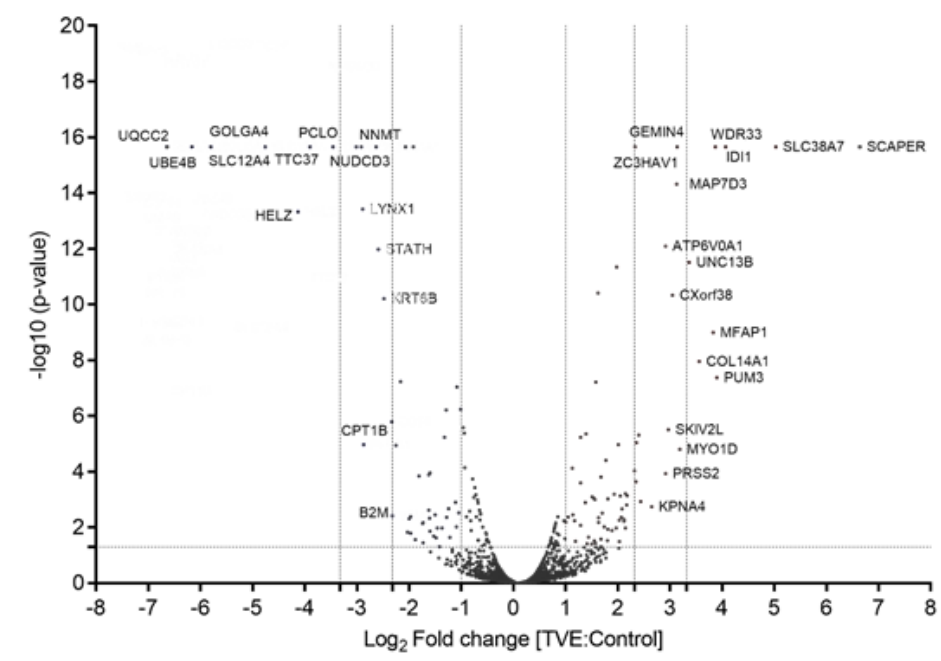

C

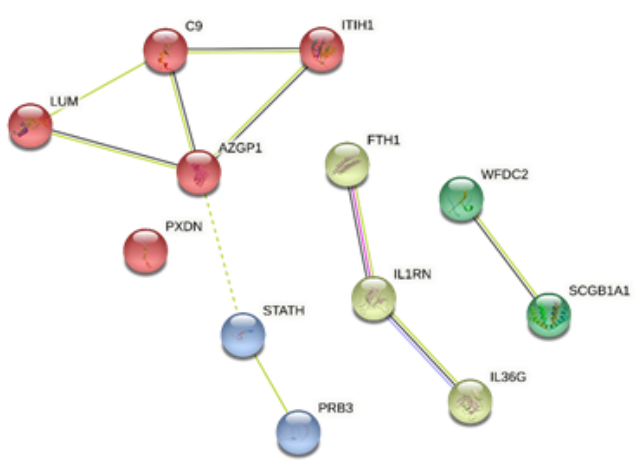

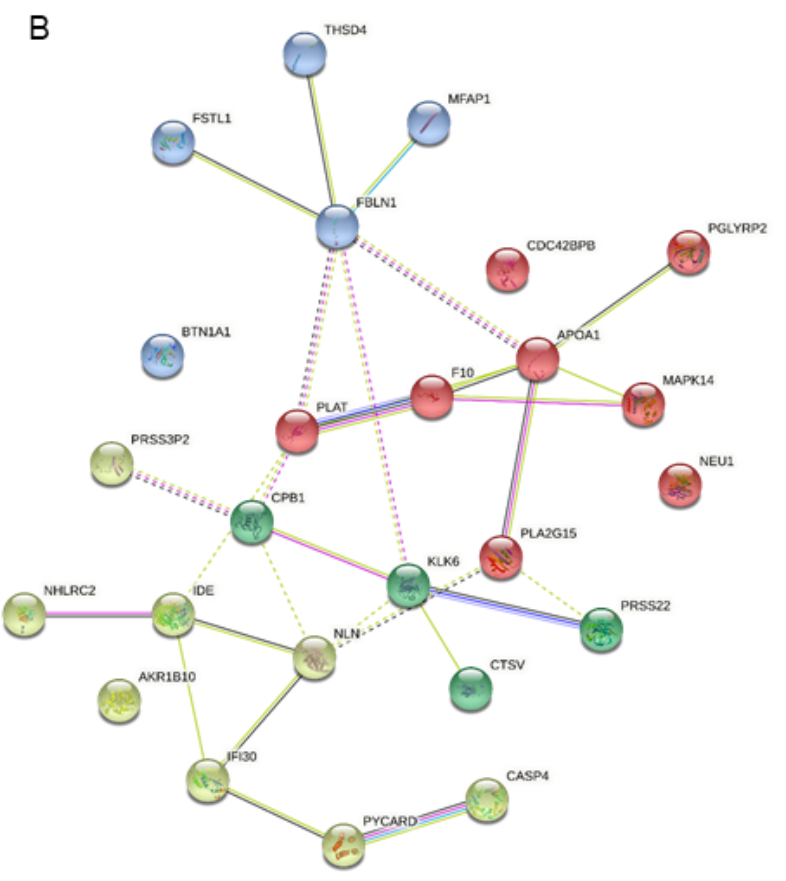


Figure 4

(A) Volcano plot of significance versus fold change of TVE vs ctrl proteins. STRING analysis of TVE more abundant (B) and less abundant (C) proteins annotated as being extracellular ones by PD. 4 different clusters are shown in different colors. Each protein is labelled through its Gene Name.

A

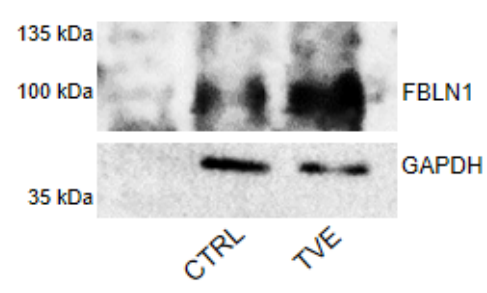

B

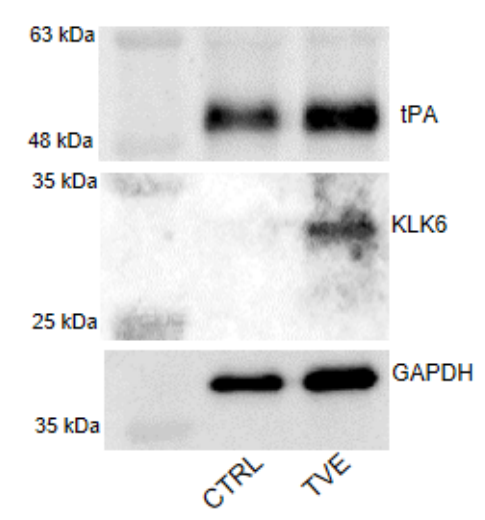

C

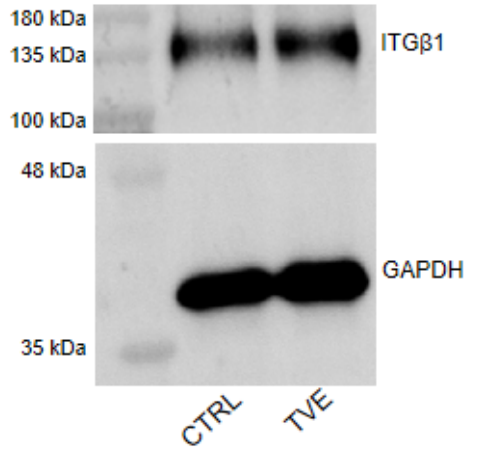

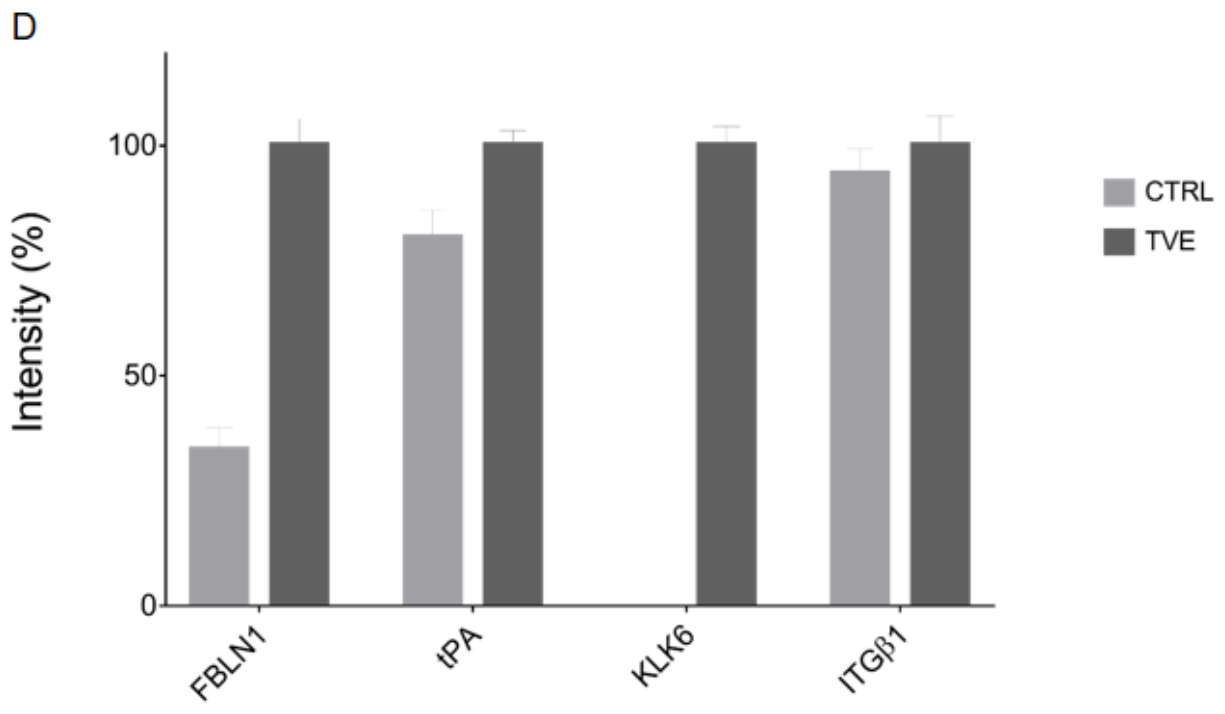

Figure 5

(A) Mass spectrometry data validation through Western Blotting for FBLN1 (A), tPA and KLK6 (B) and ITG $\beta 1$ (C), each reported with their respective GAPDH for loading normalization purposes. (D)

Densitometric analyses of panel A, B and C Western Blots. GAPDH was used as a loading normalizer and TVE intensities were set as $100 \%$.

\section{Supplementary Files}

This is a list of supplementary files associated with this preprint. Click to download.

- MorrettaetalSI.docx 